\title{
Analysis of transcripts and splice isoforms in red clover (Trifolium pratense L.) by single-molecule long-read sequencing
}

\author{
Yuehui Chao ${ }^{\dagger}$, Jianbo Yuan ${ }^{\dagger}$, Sifeng Li, Siqiao Jia, Liebao Han ${ }^{*}$ and Lixin Xü
}

\begin{abstract}
Background: Red clover (Trifolium pratense L.) is an important cool-season legume plant, which is the most widely planted forage legume after alfalfa. Although a draft genome sequence was published already, the sequences and completed structure of mRNA transcripts remain unclear, which limit further explore on red clover.

Results: In this study, the red clover transcriptome was sequenced using single-molecule long-read sequencing to identify full-length splice isoforms, and 29,730 novel isoforms from known genes and 2194 novel isoforms from novel genes were identified. A total of 5492 alternative splicing events was identified and the majority of alter spliced events in red clover was corrected as intron retention. In addition, of the 15,229 genes detected by SMRT, 8719 including 186,517 transcripts have at least one poly(A) site. Furthermore, we identified 4333 long non-coding RNAs and 3762 fusion transcripts.

Conclusions: We analyzed full-length transcriptome of red clover with PacBio SMRT. Those new findings provided important information for improving red clover draft genome annotation and fully characterization of red clover transcriptome.
\end{abstract}

Keywords: Red clover, Full-length transcript, Alternative splicing, Alternative polyadenylation, Long non-coding RNA, Fusion transcript

\section{Background}

Red clover (Trifolium pratense L.) is an important legume plant that plays a key role in sustainable intensification of livestock farming systems. Red clover is native to Europe, Western Asia and northwest Africa, but it is planted in many other regions because it is adapted to a wide range of soils $[1,2]$. As a legume plant, it has a higher protein content because of nitrogen fixation by nodulation via symbiosis with the soil microbe Rhizobium leguminarosum, and its reduced need for nitrogen fertilizer input can reduce the environmental footprint of grassland-based agriculture [3]. Red clover is a diploid $(2 \mathrm{n}=14)$ species that is naturally cross-pollinated, with a genome assembled with $309 \mathrm{Mb}$ in 39,904 scaffolds reported recently [4].

Next-generation high-throughput sequencing (NGS), also known as 2nd generation sequencing has emerged as

\footnotetext{
* Correspondence: hanliebao@163.com; liberisa@163.com

†Yuehui Chao and Jianbo Yuan contributed equally to this work.

Turfgrass Research Institute, Beijing Forestry University, Beijing 100083, China
}

a revolutionary tool to better understand differential gene expression and regulatory mechanisms. In this approach, there is no strict requirement for a reference genome sequence, so it is suitable for model or non-model species. With this tool, works on transcriptome analysis were accomplished in red clover. In 2014, RNA-Seq and analysis of red clover leaves from drought and non-drought plants showed that 6262 transcript tags were differentially expressed. The de novo assembly of a red clover transcriptome provided a rich source for gene identification, single nucleotide polymorphisms and short sequence repeats. Those RNA-Seq data supplied candidate genes for further analysis of the genetic basis of drought tolerance in red clover [5]. A de novo transcriptome assembly of red clover was used for genome-wide identification of different plant transcription factor families, gene expression analysis of different tissues and dynamic spatial gene coexpression networks [6]. As a powerful tool for description of gene expression levels and individual splice junctions, short-read RNA sequencing has been becoming focus of

(C) The Author(s). 2018 Open Access This article is distributed under the terms of the Creative Commons Attribution 4.0 International License (http://creativecommons.org/licenses/by/4.0/), which permits unrestricted use, distribution, and 
scientific researchers, but this tool cannot provide full-length sequence and alternatively spliced forms for each RNA. For example, in Arabidopsis thaliana, alternatively spliced forms exist in more than $80 \%$ multiple-exon genes [7]. Information about RNA sequence and alternative splicing forms is crucial for deeply understanding plant transcriptome and their potential biological consequences. Pacific Biosciences single molecule long reads sequencing technology (SMRT), also called the 3rd generation sequencing technology, is applied to effectively capture full-length sequence of genome and transcripts. Moreover, the new technology can accurately identify full-length splice isoforms and APA sites, and also identify a higher isoform density than reference genome. Recently, SMRT has been used to characterize the complexity of transcriptome in animals and plants [8-13]. SMRT was applied to analyze human transcriptome and about 14,000 spliced genes were identified, in which over $10 \%$ were not previously annotated [8]. PacBio SMRT was employed for whole-transcriptome profiling in Oryctolagus cuniculus and a total of 36,186 high-confidence transcripts were obtained, among which more than $23 \%$ of genic loci and $66 \%$ of isoforms have not been annotated before. Furthermore, 24,797 alternative splicing (AS) and 11,184 alternative polyadenylation (APA) events were detected, respectively [11]. The first full-length insect transcriptome was sequenced based on the PacBio platform and the first quantitative transcription map of animal mitochondrial genomes was constructed, which enriched fundamental concepts of mitochondrial gene transcription and RNA processing, particularly of the rRNA primary (sequence) structure [14]. The sorghum transcriptome was analyzed by the 3rd sequencing technology and results showed that sequencing data uncovered over 7000 novel alternative splicing events, about 11,000 novel splice isoforms, over 2100 novel genes and several thousand transcripts that differ in 3' untranslated regions due to APA [9]. Combining with NGS and SMRT, approximately $83.4 \%$ of intron-containing genes were found alternatively spliced and the results enhanced understanding on AS under normal condition and in response to ABA treatment in Arabidopsis thaliana [7]. In moso bamboo, genome-wide AS and APA were identified. The results showed that more than 42,280 distinct splicing isoforms were derived from 128,667 intron-containing full-length non-chimeric (FLNC) reads and 25,069 polyadenylation sites from 11,450 genes, 6311 of which have APA sites [12]. In wheat, a total of 91,881 high-quality FLNC reads were identified and 3,026 new genes were found not annotated previously [15]. In maize, 111,151 unique isoforms and higher isoform density than reference genome were identified with SMRT. Moreover, 867 novel high-confidence lncRNAs were identified and had a much longer mean length than those identified by Illumina short-read sequencing [10]. Those works provided useful information of transcriptomes and served as valuable resources for further research.

Although work on genome sequencing has been done in red clover, information about sequences and structure of mRNA transcripts is very limited. The genome is not well annotated and AS events, lncRNAs, splice isoforms, APA sites and fusion transcripts are largely unclear. Here we used SMRT to analyze red clover transcriptome. Compared with previous annotations, we identified 31,924 novel transcripts, 2194 of which were derived from novel genes. A total of 5492 AS events were identified and the majority of AS events was intron retention. We identified 4333 lncRNAs and the number in reference genome was 11. We also identified 3762 fusion transcripts, and fusion events were more likely to occur inter-chromosomally. The transcriptome data provided full-length sequences and gene isoforms of transcripts in red clover, which will improve genome annotation and enhance our understanding of the gene structure of red clover.

\section{Results}

\section{Red clover transcriptome sequencing with SMRT}

The transcriptome of 10 pooled samples was sequenced and analyzed with the PacBio Sequel platform to accurately capture full-length sequences and uncover full-length splice variants. RNA from pooled samples isolated and the cDNA was size-selected in fractions of lengths $<4-\mathrm{kb}$ and $>4-\mathrm{kb}$. With SMRT, a total of 7,774,277 subreads (13.24-Gb) were obtained (SRA accession: SRP149129 Additional file 1: Figure S1), with an average read length of $1703 \mathrm{bp}$ and N50 of 2,719 bp (Fig. 1a; Table 1). To provide more accurate sequence information, circular consensus sequence (CCS) was generated from reads that pass at least 2 times through the insert, and a total of 525,906 CCS were obtained (Additional file 1: Figure S1). By detecting the sequences, 437,814 were identified as full-length (containing $5^{\prime}$ primer, 3' primer and the poly(A) tail) and 434,405 were identified as full-length non-chimeric (FLNC) reads with low artificial concatemers and the mean length of FLNC was 2688 bp (Figure1b; Additional file 1: Figure S1; Table 1). The FLNC reads with similar sequences are clustered together using ICE (Iterative isoform-clustering) algorithm, and each cluster is considered as a consistent sequence with obtaining 216,028 consensus isoforms (Fig. 1c; Additional file 1: Figure S1; Table 1). Combined with non-full-length sequences, the quiver program was used to correct the consistent sequences in each cluster, resulting in 17,895 high-quality isoforms (HQs) with accuracy $>99 \%$ and 45,883 low-quality isoforms (LQs). Quiver was then used to polish the non-chimeric transcripts and then 215,586 high quality, FL, and polished consensus transcripts were generated. The mean length of polished 

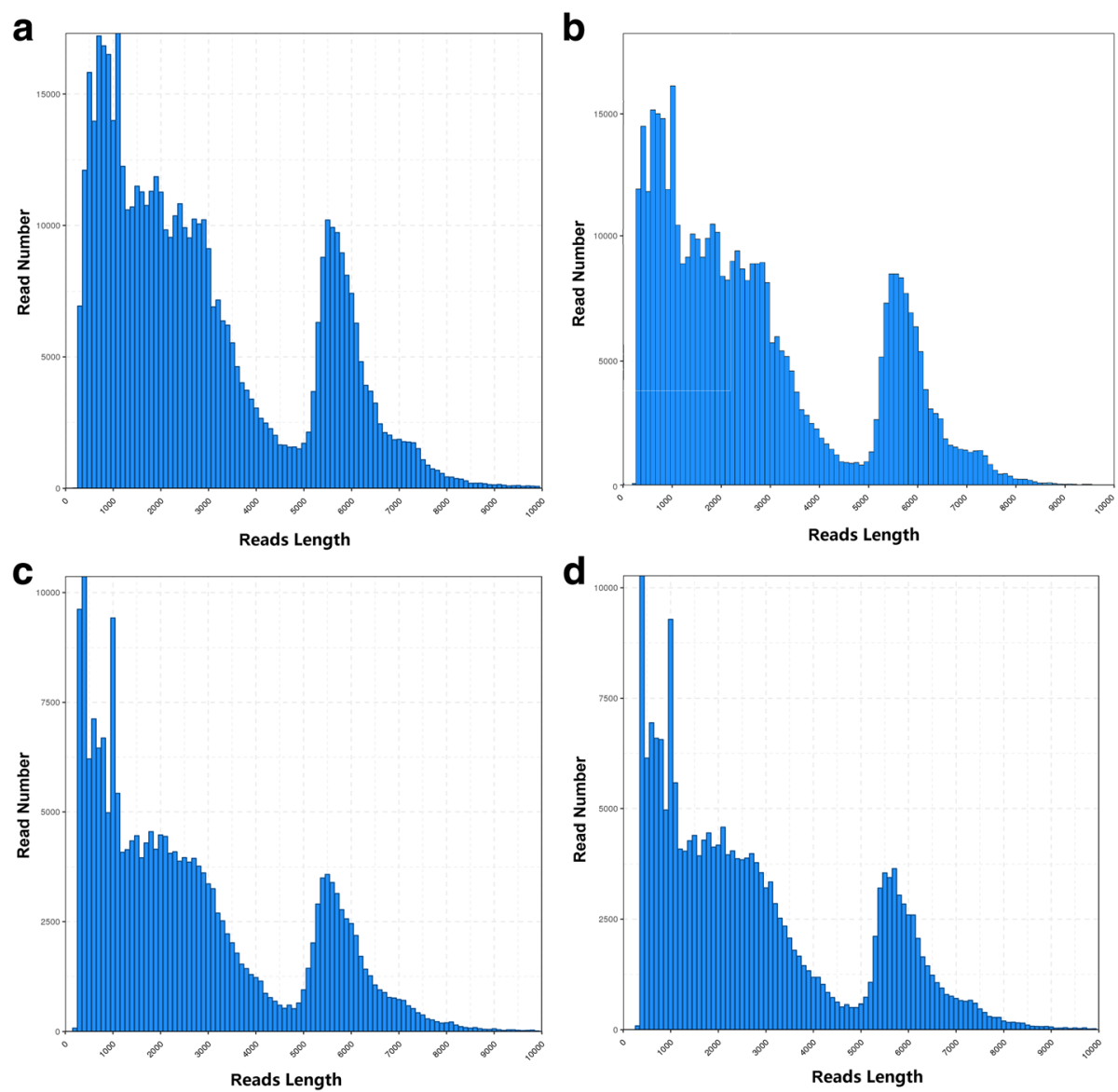

Fig. 1 Length distributions of PacBio SMRT sequencing. a Number and length distributions of 525,906 CCS sequences. b Number and length distributions of 434,405 FLNC sequences. c Number and length distributions of 216,028 consensus isoforms. $\mathbf{d}$ Number and length distributions of 206,465 corrected isoforms

consensus isoforms was $2657 \mathrm{bp}$, with N50 of $4616 \mathrm{bp}$ (Table 1). All polished consensus isoforms were corrected using the NGS reads with Proovread software [16], resulting in 206,465 corrected isoforms (Additional file 1: Figure S1), with a N50 length of $4738 \mathrm{bp}$ and mean read length of $2789 \mathrm{bp}$. In total, 189,960 isoforms (92.0\%) were longer than $500 \mathrm{bp}$, and 155,606 isoforms $(75.4 \%)$ were longer than 1-kb (Figure 1d; Table 1).

\section{Functional annotation of transcripts}

All 206,465 transcripts (corrected isoforms) were functional annotated by searching NR, Swissprot, GO, COG, KOG, Pfam and KEGG databases and a total of 199,093 transcripts (96.4\%) was annotated (Fig. 2a; Additional file 2:
Table S1). We analyzed homologous species by comparing the transcript sequences to the NR database, and the results showed that the largest five number of transcripts was distributed in Trifolium subterraneum (84,418), Medicago truncatula $(57,646)$, Cicer arietinum $(17,825)$, Trifolium pratense $(10,284)$ and Glycine max (1726) (Fig. 2b). GO analysis showed that the enrichment of 80,667 transcripts could be divided into three groups, including biological processes, molecular functions and cellular components. Genes involved in biological processes consisted of metabolic, cellular, single-organism, localization, biological regulation, signal processes and so on (Fig. 2c). Genes in the molecular function were mainly enriched for binding, catalytic, transporter, structural molecular and

Table 1 Summary of reads from PacBio single-molecule long-read sequencing

\begin{tabular}{|c|c|c|c|c|c|c|}
\hline & Subread & CCS & FLNC & ICE consensus & Polished consensus & Correct Consensus \\
\hline Number & $7,774,277$ & 525,906 & 434,405 & 216,028 & 215,586 & 206,465 \\
\hline Mean Length & 1703 & 2882 & 2688 & 2638 & 2657 & 2789 \\
\hline N50 & 2719 & 5026 & 4840 & 4591 & 4616 & 4738 \\
\hline
\end{tabular}




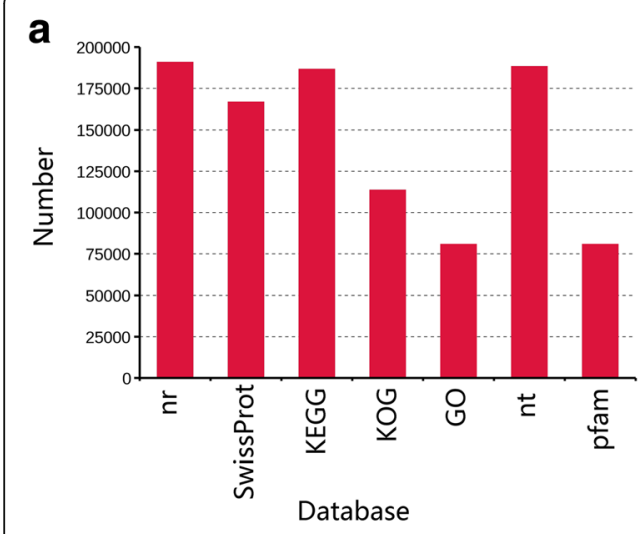

C

Gene Function Classification (GO)

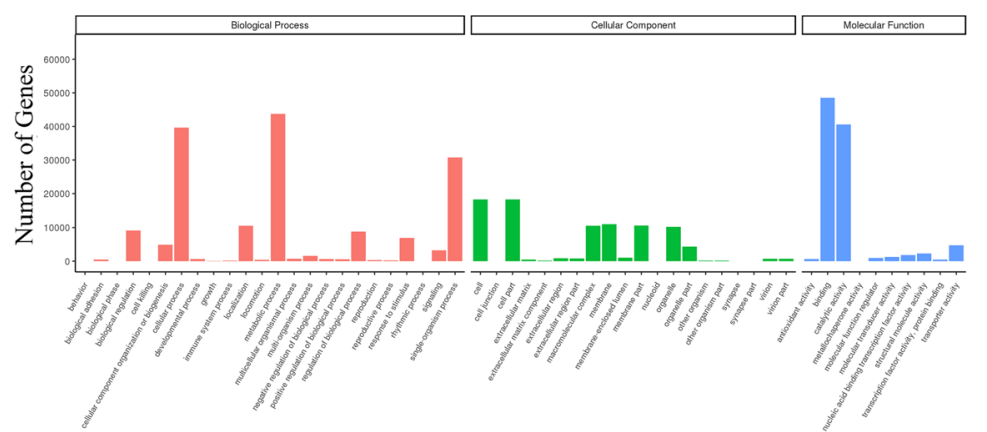

b

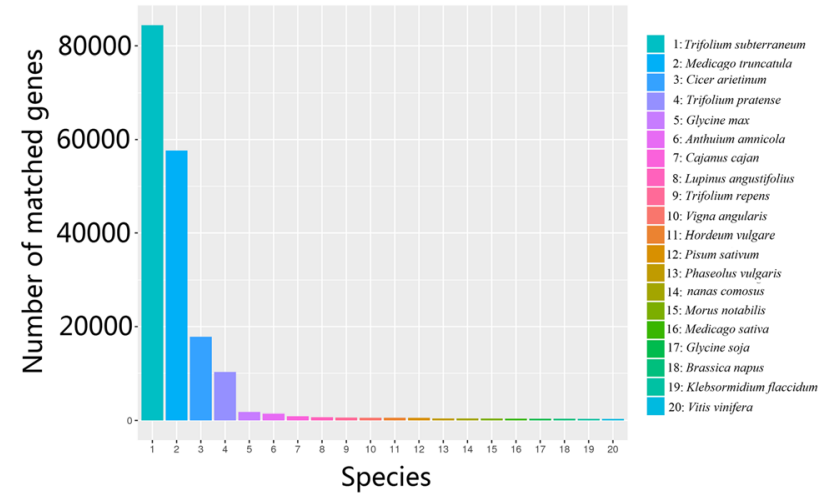

d

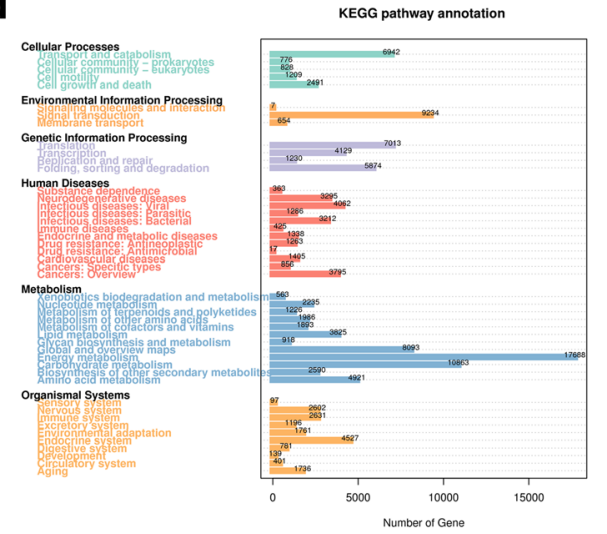

Fig. 2 Function annotation of corrected isoforms. a Function annotation of transcripts in all databases. Nr, Non-Redundant Protein Database; GO, Gene Ontology; COG/KOG, Cluster of Orthologous Groups of proteins; KEGG, Kyoto Encyclopedia of Genes and Genomes. b Nr Homologous species distribution diagram of transcripts. c Distribution of GO terms for all annotated transcripts in biological process, cellular component and molecular function. d KEGG pathways enriched of transcripts

nucleic acid binding transcription factor activities. For the category "Cellular Component", genes were mainly involved in cell, cell part, membrane, organelle, membrane part and others. The KEGG results demonstrated that 186,497 transcripts were mapped to 368 KEGG pathways (Fig. 2d).

\section{Genome mapping}

We compared all 206,465 corrected isoforms against the draft genome sequence of red clover using GMAP. A total of 136,395 reads $(66.06 \%)$ were mapped to the reference genome (Additional file 1: Figure S1). Based on the mapped results, these reads could be divided into four groups (Fig. 3a). Unmapped, consisted of 70,070 reads (33.94\%) with no significant mapping to the draft genome. Multiple mapped, contained 130,525 reads (63.22\%) showing multiple alignments. Mapped to ' + ', included 4414 reads were mapped to the positive strand of the genome and mapped to '-', included 1456 reads were mapped to the opposite strand of the genome. Isoforms spanning two or more genes are removed from downstream splice isoform analysis; however, they likely represent misannotations in the gene models. In this case, 48,626 isoforms were removed, and the remaining 87,769 transcripts were then assembled into clusters, resulting in 39,787 isoforms. By analysis, $89.2 \%$ of those reads aligned to 13,284 annotated genes out of 40,868 genes in the reference genome.

\section{Novel genes and transcripts finding}

All FLNC sequences were compared against the genome sequence with GMAP, and 200,787 reads (46.22\%) were mapped to the reference genome. Mapped FLNC were divided into 3 types: 1) 98,030 same in reference genome; 2) 95,898 novel isoforms from known genes; 3) 6859 novel isoforms from novel genes (Fig. 3b). We also compared 39,787 isoforms against the reference genome, and $89.2 \%$ of those reads aligned to 13,284 annotated genes out of 40,868 genes in the reference genome. We identified 7863 same in reference genome, 29,730 novel isoforms from known genes and 2194 novel isoforms from novel genes (Fig. 3c; Fig. 4).

\section{CDS finding and exon/intron structure analysis}

Coding sequences were identified by ANGEL software, resulting in 36,622 coding sequences with a mean length 


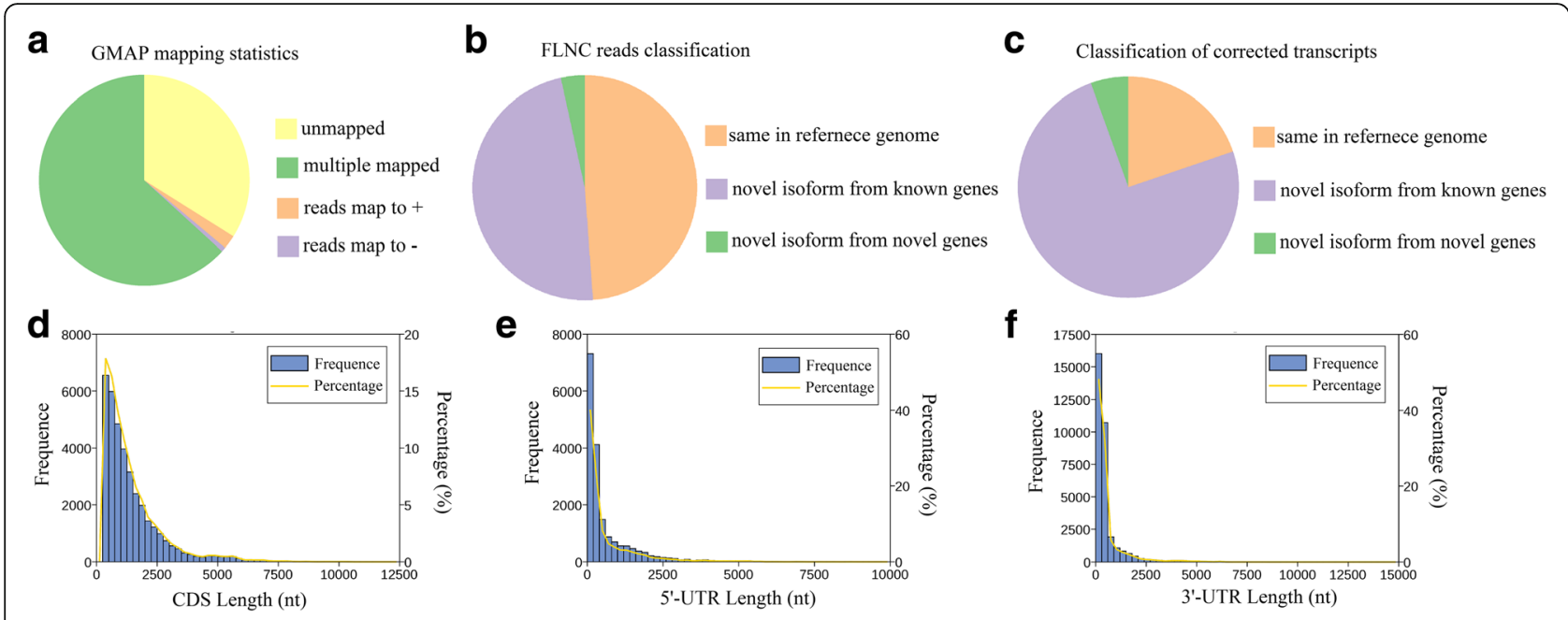

Fig. 3 GMAP analysis and CDS-UTR structures of SMRT sequences. a GMAP analysis of corrected reads to reference genome. $\mathbf{b}$ Classification of FLNC sequences mapped to reference genome. c Classification of corrected isoforms mapped to reference genome. $\mathbf{d}$ Number, percentage and length distributions of coding sequences of corrected isoforms. e Number, percentage and length distributions of $5^{\prime}$-UTR of corrected isoforms. $\mathbf{f}$ Number, percentage and length distributions of $3^{\prime}-U T R$ of corrected isoforms

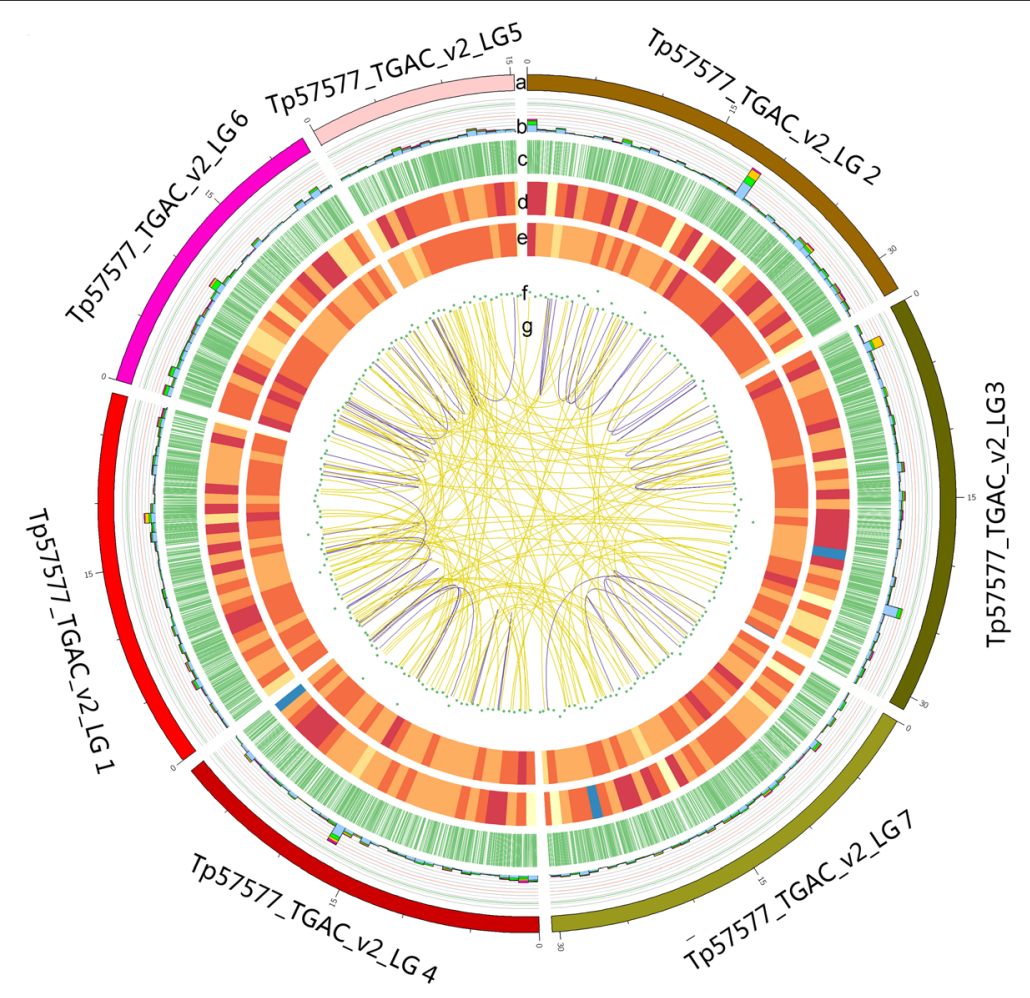

Fig. 4 CIRCOS visualization of Iso-Seq data at the genome-wide level. a Karyotype of red clover chromosomes. b Comparison of APA sites mapped to red clover reference genome. c Novel transcript density from Iso-Seq data. The closer to red, the higher density the color represents and the closer to blue, the lower density the color represents. $\mathbf{d}$ Novel gene density from Iso-Seq data. e LncRNA density. The closer to the center, the lower density the dot represents. $\mathbf{f}$ Linkage of fusion transcripts. Purple, intra-chromosomal; Yellow, inter-chromosomal 
of 1435.26 nucleotides (Fig. 3d). Sequences with start and stop codons were defined as complete coding sequences, and 10,304 carried complete ORFs (Table 2). The number and length distributions of $5^{\prime}$ and $3^{\prime}$ UTRs were investigated. The results displayed 18,198 5' UTRs with a mean length of $650.5 \mathrm{bp}$ and 33,219 3' UTRs with a mean length of $559.45 \mathrm{bp}$ (Fig. 3e and f).

By SMRT, 39,787 transcripts were used to analyze exon structure. One exon was found in 8302 transcripts (20.87\%) and the transcript number with 2 exons was 5175 (13.01\%), while transcripts with more than 20 exons was 1927 (4.84\%) (Fig. 5a; Additional file 3: Table S2). In reference genome, the transcripts number with 1 exon was 40,868 (49.18\%) and transcripts with more than 20 exons was 7693 (9.26\%) (Fig. 5b). The intron number was 596,960 in reference genome with 14.61 introns per gene (intron/gene) and that was 247,837 in our Iso-Seq data with 16.27 intron/gene. We further analyzed the median number of introns in intron containing genes, and the result showed the number is five in red clover, which is four in Arabidopsis thaliana.

\section{AS and splice isoforms in red clover}

AS events in red clover were analyzed with Suppa software. We detected a total 5492 AS events from the Iso-Seq reads and there are $1123 \mathrm{AS}$ events in reference genome. The majority of AS events in Iso-Seq was intron retention with similar distribution to other plants, while the majority of AS events in reference genome was alternative 3' splice (Fig. 4; Fig. 5c). Compared with reference genome, 4831 AS events occurred specifically in 1996 genes based on Iso-Seq data (Additional file 4: Table S3). Those AS events in our study largely enriched transcripts information in the draft version of the red clover genome.

In reference genome, there are 40,868 genes annotated and 39,729 genes were shown to have 2 isoforms. The largest number of isoforms was 6 found in 3 genes, Tp57577_TGAC_v2_gene16492 (Tp57577_TGAC_v2_LG7: 11814616-11,818,691), Tp57577_TGAC_v2_gene3878 (Tp 57577_TGAC_v2_LG6: 17447258-17,473,786) and Tp5757 7_TGAC_v2_gene39688 (Tp57577_TGAC_v2_scaf_1548: 1 1427-14,327). In our Iso-Seq analysis, only one single isoform was detected in 6904 genes and two or more isoforms were found in 8325 genes. Six and more than 6 splice isoforms were detected in 1329 genes (Fig. 5d; Additional file 5: Table S4). For example, six isoforms were detected in Tp57577_TGAC_v2_gene25740 based on Iso-Seq, while

Table 2 CDS identification from PacBio single-molecule long-read sequencing

\begin{tabular}{cccccc}
\hline & CDS & Completed CDS & 3' partial & 5' partial & Uncertain \\
\hline Number & 36,622 & 10,304 & 1929 & 13,158 & 11,231 \\
\hline
\end{tabular}

the isoform number was 3 in the red clover annotation (Fig. 5e). The average number of isoforms per gene (2.61) identified from Iso-Seq data were significantly more than that (2.03) in the reference genome. The largest number of isoforms were 113 found in Tp57577_TGAC_v2_gene14962 (Tp57577_TGAC_v2_scaf_591:48683-68,918) encoding the chromatin structure-remodeling complex protein. To further validate the AS events and isoforms, we randomly chose 5 candidates and primers were designed based on the sequences of Iso-Seq. The candidate genes were validated by RT-PCR and Sanger sequencing (Additional file 6: Figure S2).

\section{Identification of IncRNA, fusion transcript and TF}

A total of 4333 lncRNAs were identified by three methods and $3050(70.39 \%)$ of the lncRNAs were single exon (Figure 6a, b and c; Additional file 7: Table S5). We classified them into 4 groups: 1148 lincRNA (26.49\%), 289 antisense (6.67\%), 1747 sense intronic $(40.32 \%)$ and 1149 sense overlapping lncRNAs (26.52\%) (Fig. 6b). BLASTN was used to get rid of the previously discovered 11 lncRNAs downloaded from ensemble website, and all predicted lncRNA (4333) were identified as novel lncRNAs with a mean length of 665.39 bp (Fig. 6c and d). Mapping lncRNAs to chromosomes was shown in Fig. 4, and identification of lncRNAs enriched genome information of red clover.

In reference genome, a total of 39,051 scaffold sequences was used to predict fusion transcript. In this study, with illumina short reads, we identified 3762 fusion transcripts out of 39,051 scaffold sequences (Additional file 8: Table S6), and fusion events were more likely to occur inter-chromosomally (3665) than intra-chromosomally (97). Seven scaffolds, assembled to chromosome level from 39,051 scaffold sequences, were picked up for fusion transcript identification. We also found 334 fusion transcripts, including 238 inter-chromosomal and 96 intra-chromosomal sequences (Fig. 4). To further validate the fusion transcripts, we randomly chose 10 candidates and primers were designed based on the sequences of Iso-Seq. Eight (80\%) were validated by RT-PCR and Sanger sequencing (Additional file 9: Figure S3). For example, i0_HQ_c62675/f4p0/1006 was fused by two gene TP57577_TGAC_V2_GENE20501 and TP57577_TGAC_V2_GENE12817 (Additional file 8: Table S6). The PCR products were confirmed by Sanger sequencing and the results proved the authenticity of these chimeric RNAs.

Transcript factors (TFs) and transcript regulators (TRs) play important regulatory roles in plant growth and development. They were identified and classified with iTAK and 2302 putative TF (1361) and TR (941) members from 89 families were identified (Additional file 10: Table S7). The top 29 families identified were shown in Fig. 7a. We compared those members against 2065 known TFs from 


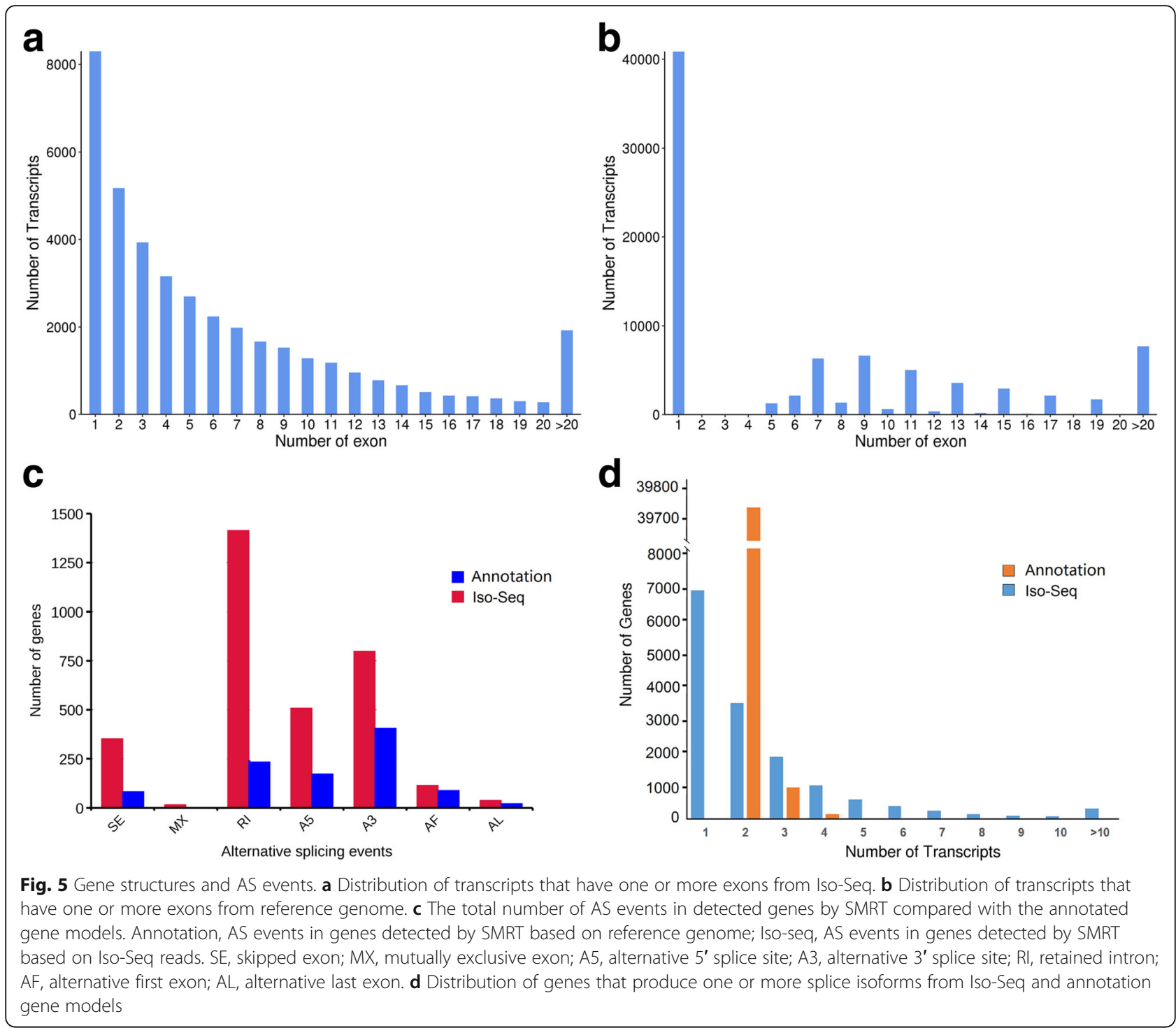

red clover, and the result showed that 249 were identified as known and 2053 were identified as novel.

\section{APA analysis}

To identify accurately differential polyadenylation sites in red clover, 3' ends of transcripts from Iso-Seq were investigated. Of the 15,229 genes detected by Iso-Seq, 8719 including 186,517 transcripts have at least one poly(A) site, and 497 genes have at least five poly(A) sites (Fig. 4; Fig. 7b; Additional file 11: Table S8). The average number of poly(A) site per gene was 1.97 . We analyzed the nucleotide composition in the upstream (50 nts) and downstream (50 nts) of all poly(A) cleavage sites for nucleotide bias. Nucleotide composition flanking all poly(A) sites was analyzed. Consistent with findings in Sorghum bicolor and Phyllostachys edulis, we observed clear nucleotide bias around poly(A) sites in red clover with an enrichment of uracil (U) upstream and adenine (A) downstream of the cleavage site in 3'-UTRs (Fig. 7c). To identify potential cis-elements necessary for polyadenylation, we performed a MEME analysis for motifs enriched upstream of the cleavage site using 50 nucleotides upstream from the predominant poly(A) site of all transcripts. One conserved motif (AAUAAA) was identified upstream of poly(A) cleavage sites (Fig. 7d), and this motif was consistent with previous reported patterns in Sorghum bicolor, maize and Phyllostachys edulis $[9,12,17]$.

\section{Discussion}

By now, the draft genome sequence had been published, but the transcriptome was not fully explored. The full-length mRNA sequences, alterative spliced 
a

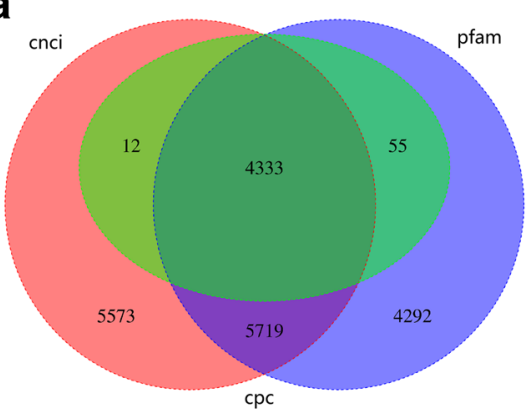

C

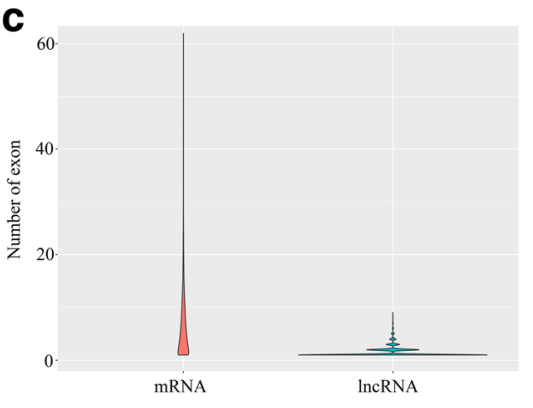

b

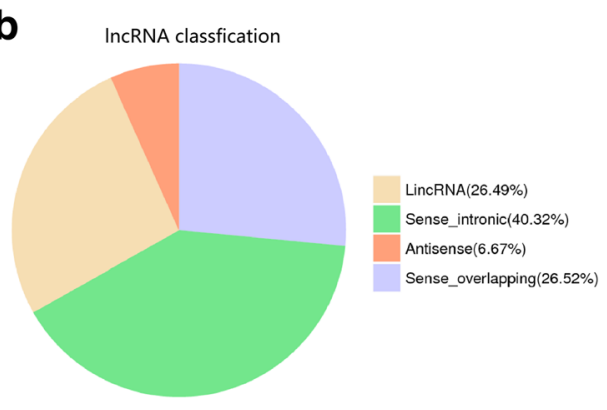

d

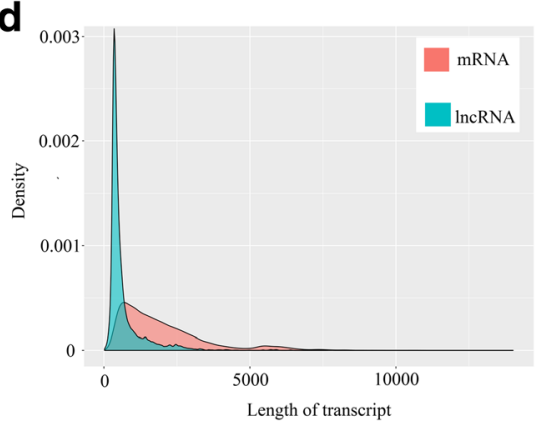

Fig. 6 Identification of IncRNAs. a Venn diagram of IncRNAs predicted by CPC, CNCI and pfam methods. b Proportions of four types of IncRNA. c Comparison of exon number of mRNA and IncRNAs identified in this study. $\mathbf{d}$ Density and length distributions of mRNAs and IncRNAs in red clover
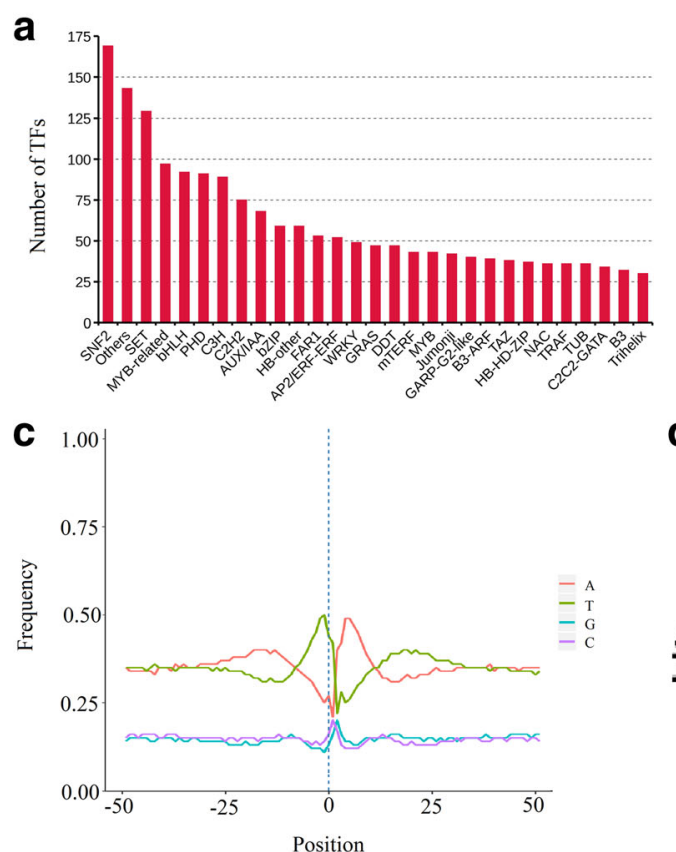

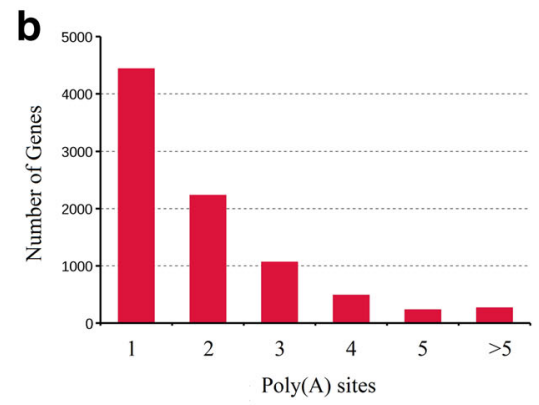

d

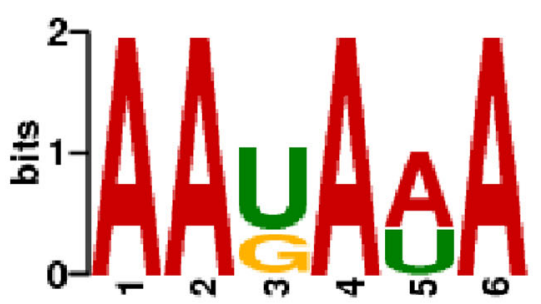

Fig. 7 TF prediction and APA analysis. a Number and family of top 29 TFs predicted by SMRT. b Distribution of the number of poly(A) sites per gene. $\mathbf{c}$ Nucleotide composition around poly $(A)$ cleavage sites. The relative frequency of a nucleotide is shown as a function of genomic position across all poly(A) cleavage sites detected in our data. $\mathbf{d}$ MEME analysis of an over-represented motif at 25-nts upstream of the poly(A) site in red clover transcripts 
transcripts, APA sites and fusion transcripts in red clover remains unknown. With development of sequencing technology, single molecule long reads sequencing technology provides new insights into full-length sequence, alterative splice, gene structure and APA. In this work, analyzed full-length transcriptome of red clover with PacBio SMRT. PacBio sequencing yielded 525,906 CCS, in which 434,405 are identified as FLNC transcripts. The length of the FLNC sequence reflects the length of the cDNA sequence in each sequencing library, and the library can be assessed by the length of the FLNC sequence. The length of the FLNC sequence is consistent with the size of the library (Figure1). A total of 216,028 consensus isoforms were generated with ICE algorithm and 215,586 polished consensus were identified. Combined SMRT with NGS, 206,465 corrected consensus reads were obtained in total. Meanwhile, 5492 AS events, 4333 lncRNAs, and 3762 fusion transcripts were identified and 8719 genes with 186,517 transcripts have at least one poly(A) site. Those new findings provided important information for improving red clover draft genome annotation and fully characterization of red clover transcriptome.

Full-length transcript sequence information is very useful for both genome annotation and gene function studies in plants. Our results demonstrated that PacBio sequencing is an effective technology for obtaining reliable full-length transcript sequence information in red clover. PacBio sequencing yielded 525,906 CCS, $82.6 \%$ of which are identified as FLNC transcripts. PacBio CCSs and FLNC reads completely avoided the need to assemble short NGS reads. With NGS data, 206,465 corrected isoforms were obtained, and $75.4 \%$ were longer than 1 -kb. About 10,304 isoforms were found to carry completed ORF. The high capacity of PacBio transcriptome sequencing to generate full-length transcript sequence information may well be related to its long-read property. In our work, 15,229 genes were detected by SMRT with a mean of $3788 \mathrm{bp}$, which is $405 \mathrm{bp}$ larger in size than that in reference genome. Previous reports supplied similar suggestion, showing that newly discovered transcripts by SMRT in wheat were on average more than 45 bp longer than the known transcripts [15]. The new sequencing technology enriches transcript resources and provides advantages for discovering novel or uncharacterized transcript isoforms and genes. In this work, we identified 29,730 novel isoforms from known genes and 2194 novel isoforms from novel genes in the draft genome based on SMRT data. Those findings not only enrich the transcriptional information of the draft genome sequence but also are useful for functional studies of important genes in further research.

Previous studies on red clover transcriptome mainly focused on NGS technology to exploring gene discovery, differential expression genes analysis, pathways enrichment, marker identification and so on $[5,6]$. But this tool is often unable to accurately capture or assemble full-length transcripts. Based on PacBio SMRT, fragmenting of RNA is not required and intact transcript sequence information is provided by avoiding assembly. In our work, we characterized red clover transcriptome with PacBio SMRT. The N50 and average lengths of contigs assembled from previous project were 1707 and $1262 \mathrm{bp}$ [6], and those were much larger from Iso-seq (Table 1). Those results showed that SMRT has a better capacity in capturing transcript sequences, especially long transcript sequences. As well as full-length transcripts, the AS event identification is another advantage. We detected a total 5492 AS events from the Iso-Seq reads and there are 1123 AS events in reference genome. The majority of AS events in Iso-Seq is intron retention with similar distribution to maize [10], bamboo [12], Amborella trichopoda [18], strawberry [19] and so on, while the majority of AS events in reference genome is alternative $3^{\prime}$ splice. Those AS events in our study largely enriched transcripts information in the draft version of the red clover genome. But we did not analyze the isoform expression levels in current project, so detailed expression levels of isoforms derived from one gene in red clover should be analyzed combined expression analysis from NGS with AS isoforms from SMRT in the future.

In our study, we found 3762 fusion transcripts, and fusion events were more likely to occur inter-chromosomally than intra-chromosomally. The chimeric fusion events in red clover enhanced the complexity of the red clover transcriptome and those results were consistent with the higher proportion of inter-chromosomal to intra-chromosomal fusions in maize [10]. To confirm the fusion transcripts, we randomly selected 10 candidates to design primers for RT-PCR analysis. As predicted, 8 candidates were validated by RT-PCR and Sanger sequencing. The findings in fusion transcript, would greatly improves draft version of gene models in red clover. LncRNAs, a hotpot of molecular biology, is thought to be important regulators with function little known. In this study, we identified 4333 lncRNAs with a mean length of $665.39 \mathrm{bp}$ and the largest type in number is sense intronic. We downloaded 11 known IncRNA from ensemble website, the mean length is $93.09 \mathrm{bp}$. By comparison, the newly identified lncRNAs were much longer, with a mean length 7-time larger than that of known lncRNAs. Those results is similar to the former study. In maize, IncRNAs identified by SMRT, most of which are intergenic, are much longer than those previously described, and some of which are as long as 6-kb [10]. Of the 15,229 genes detected by Iso-Seq, 8719 including 186,517 transcripts have at least one poly(A) site (Fig. 4; Fig. 7b). This work provides useful information for future analysis of the relation of APA and gene function. 


\section{Conclusions}

In conclusion, we analyzed full-length transcriptome of red clover with PacBio SMRT. Those new findings provided important information for improving red clover draft genome annotation and fully characterization of red clover transcriptome.

\section{Methods}

\section{Plant samples}

Red clover seeds (cv. Common) received as gifts from Top Green Group (Beijing, China) were sown in nutrition medium containing peat, vermiculite and pearlite (3:3:1) until germination, and then plants were grown at Beijing Forestry University $\left(\mathrm{E} 116^{\circ} 20^{\prime}\right.$; N40 $\left.00^{\prime}\right)$ under greenhouse conditions at $25 / 23^{\circ} \mathrm{C}$ (day/night) with a 16 -h photoperiod in growth chambers. Different tissues, including leaves, stems, roots and flowers of 1-year-old plants were harvested in May 2017. To obtain more spliced isoforms, red clover plants were stressed by $100 \mathrm{mM} \mathrm{NaCl}$ or $10 \%$ PEG for 1 day, or induced by $10 \mu \mathrm{M} A B A, 10 \mu \mathrm{M} \mathrm{GA}_{3}$, $10 \mu \mathrm{M}$ IAA and $10 \mu \mathrm{M} 6$-BA for $6 \mathrm{~h}$. All samples were harvested and frozen in liquid nitrogen and stored at -80 ${ }^{\circ} \mathrm{C}$ for further experiments.

\section{Library preparation and SMRT sequencing}

Total RNA from each sample was isolated using a Plant RNA kit (Omega bio-Tech, USA) and then treated with RNase-free DNase I (NEB) to remove contaminated genomic DNA. RNA degradation and contamination was monitored on $1 \%$ agarose gels and RNA purity was checked using the NanoPhotometer ${ }^{\bullet}$ spectrophotometer (IMPLEN, CA, USA). RNA concentration was measured using Qubit ${ }^{\circ}$ RNA Assay Kit in Qubit $^{\circ}$ 2.0 Flurometer (Life Technologies, CA, USA) and RNA integrity was assessed using the RNA Nano 6000 Assay Kit of the Bioanalyzer 2100 system (Agilent Technologies, CA, USA). A total amount of $5 \mu \mathrm{g}$ of total RNA (equally mixed with all RNAs) was used as input into the Clontech SMARTer reaction. Size fractionation and selection $(<4 \mathrm{~kb}$ and $>4 \mathrm{~kb})$ were performed using the BluePippin ${ }^{\text {tw }}$ Size Selection System (Sage Science, Beverly, MA). Two SMRT bell libraries were constructed with the Pacific Biosciences DNA Template Prep Kit 2.0 and SMRT sequencing was then performed on the Pacific Bioscience Sequel System.

\section{Quality filtering and error correction}

Raw reads was processed using the SMRTlink (version 4.0) software with parameters: minLength $=200$, minReadScore $=0.75$. CCSs were generated from subread BAM files (parameters: min_length 200, max_drop_fraction 0.8, no_polish TRUE, min_zscore - 999, min_passes 1 , min_predicted_accuracy 0.8 , max_length 18,000 ) and a CCS.BAM file was output. By identifying the $5^{\prime}$ and 3 ' adapters and the poly(A) tail, CCS were then classified into full length and non-full length reads. A full-length read contained both the $5^{\prime}$ and $3^{\prime}$ primers and there was a poly(A) tail signal preceding the 3 ' primer. CCS with all three elements and not containing any additional copies of the adapter sequence within the DNA fragment are classified as FLNC. Then, consensus isoforms were identified using the algorithm of ICE (Iterative Clustering for Error Correction) from FLNC and were further polished with non-full length reads to obtain high-quality isoforms with post-correction accuracy above 99\% using Quiver (parameters: hq_quiver_min_accuracy 0.99 , bin_by_primer false, bin_size_kb 1 , qv_trim_5p 100, qv_trim_3p 30). The Illumina RNA-Seq data (SRA submission number: SUB2425623) generated by our lab was used to correct nucleotide indels and mismatches in consensus reads with the software Proovread (Version 2.12) [16], resulting in corrected isoforms.

\section{Mapping to the reference genome and gene structure analysis}

FLNC and corrected isoforms were aligned to the reference genome with the Genome Mapping and Alignment Program (GMAP, version: 2017-01-14) [20] with parameters: --no-chimeras, --cross-species, --expand-offsets 1 -B 5 -K 50000 -f samse $-n$. The output fies are in the BAM format. Gene structure analysis was performed using TAPIS pipeline (Version 1.2.1, https://bitbucket.org/ comp_bio/tapis) [9]. The gff3 format genome annotation file was transfer into GTF format with gffread [21] and then used for gene and transcript determination. Alternative splicing events were identified using the SUPPAA (Version: 2017-02-07) [22]. SUPPA generates different alternative splicing event types: exon skipping (SE), alternative $5^{\prime}$ and $3^{\prime}$ splice sites (A5/A3), mutually exclusive exons (MX), intron retention (RI), and alternative first and last exons (AF/AL). The exon-intron structure for each transcript were predicted and the numbers of introns were statistically analyzed in a transcriptome level. APA events were then analyzed by TAPIS described previously [9]. To identify poly(A) sequence signals in Iso-Seq data, MEME analysis was performed on the sequence of 50 nucleotides upstream of the 186,517 poly(A) sites. MEME-ChIP was run locally on a Linux system described previously [9]. Fusion transcripts were determined as transcripts mapping to two or more long-distance range genes and was validated by at least two Illumina reads described as previously [10].

\section{Functional annotation}

Corrected isoforms were searched against NCBI non-redundant (NR), NCBI nucleotide sequence (NT), Swiss-Prot, Cluster of Orthologous Groups (KOG/COG) [23] and Kyoto Encyclopedia of Genes and Genomes (KEGG, version 58) [24] databases with BLAST software 
(version 2.2.26) under a threshold E-value $\leq 10-5$. Gene Ontology (GO) annotations were determined based on the best BLASTX hit from the NR database using the Blast2GO software (version 2.3.5, E-value $\leq 10-5$ ) [25]. KEGG pathway analyses were performed using the KEGG Automatic Annotation Server (KAAS1) and HMMER software [26] was used to search Pfam database.

\section{Identification of ORFs, TFs and IncRNAs}

Corrected isoforms were used for further analysis. To predict ORFs, the ANGEL pipeline, a long read implementation of ANGLE, was used to find potential coding sequences from transcripts [27]. Those transcripts containing complete ORFs as well as 5'- and 3'-UTRs (untranslated regions) were designated as full-length transcripts and the putative protein sequences were predicted. The transcription factors were predicted with iTAK software [28] from putative protein sequences. A total of 2065 known red clover TFs were downloaded from PlantTFDB (Plant Transcription Factor Database v4.0) [29], and blastp was ran with the following cutoff criteria: E-value $\leq 10-5$, min-coverage $=85 \%$ and min-identity $=90 \%$. The red clover lncRNAs were downloaded from ensemble website (ftp://ftp.ensemblgenomes.org/pub/plants/release-37/fasta /trifolium_pratense/ncrna/) and all 11 known lncRNAs were identified as sense intronic type. To identify long non-coding RNA (lncRNA) in the PacBio data, three analysis methods including CPC [30], CNCI [31] and Pfam [32] were used and then transcripts with length less than 300 bp predicted in all 3 methods were removed. BLASTN was used to get rid of the previously discovered lncRNAs under a criteria of e-value $\leq 1 \mathrm{e}-10$, min-identity $=90 \%$ and min-coverage $=85 \%$. The lncRNAs were divided into four groups: lincRNA, antisense, senese intronic and sense overlapping based on the method reported by Mathew [33].

\section{RT-PCR validation of fusion transcripts}

For PCR validation of fusion transcripts, gene-specific primers were designed using DNA Man (Version 6.0) and Primer Premier (Version 5.0). All primers used in the RT-PCR analyses are reported in Additional file 12: Table S9.

\section{Additional files}

Additional file 1: Figure S1. Flow chart of bioinformation analysis. (DOCX $241 \mathrm{~kb}$ )

Additional file 2: Table S1. Function annotation of all corrected isoforms by SMRT. (XLSX $6767 \mathrm{~kb})$

Additional file 3: Table S2. Exon number and chromosome location of genes and isoforms detected by SMRT. (XLSX $2109 \mathrm{~kb}$ )

Additional file 4: Table S3. AS analysis of genes and isoforms by SMRT. (XLSX $382 \mathrm{~kb}$ )
Additional file 5: Table S4. Number of splice isoforms in genes by SMRT. (XLSX 798 kb)

Additional file 6: Figure S2. RT-PCR validation of AS and isoforms in 5 candidate genes. Arrows, PCR products; M, DNA Marker DL2000 Plus II; 1 , Novelgene0860; 2, Tp57577_TGAC_v2_gene10390; 3,

Tp57577_TGAC_v2_gene11337; 4, Tp57577_TGAC_v2_gene11508; 5, Novelgene0380. (DOCX $228 \mathrm{~kb})$

Additional file 7: Table S5. Exon number of mRNAs and IncRNAs from Iso-Seq. (XLSX 1677 kb)

Additional file 8: Table S6. Information of fusion transcripts from Iso-Seq. (XLSX 527 kb)

Additional file 9: Figure S3. RT-PCR validation of 10 fusion transcripts. M, DNA Marker; 1, i0_HQ_c62675/f4p0/1006; 2, i3_LQ_c25970/f1 p0/3394; 3, i2_HQ_c22922/f2p46/2488; 4, i1_LQ_c86101/f1p0/1505; 5,

i2_LQ_c40661/f1 p0/2186; 6, i2_LQ_c60404/f1 p0/2058; 7, i0_LQ_c129257/ f1p0/896; 8, i0_LQ_c8517/f3p0/608; 9, i1_HQ_c82285/f12p0/1057; 10, i1_LQ_c24613/f1p2/1285. (DOCX 687 kb)

Additional file 10: Table S7. Transcription factor prediction of Iso-Seq reads. (XLSX $82 \mathrm{~kb})$

Additional file 11: Table S8. APA sites of genes detected by SMRT. (XLSX $401 \mathrm{~kb}$ )

Additional file 12: Table S9. Primers used for RT-PCR validation. (XLSX $19 \mathrm{~kb})$

\section{Abbreviations}

APA: Alternative polyadenylation; AS: Alterative splice; CCS: Circular consensus sequence; FLNC: Full-length non-chimeric; LncRNA: Long noncoding RNA; ORF: Open reading frames; TF: Transcript factor; TF: Transcript factor; TR: Transcript regulator

\section{Acknowledgements}

We thank Dr. Feifei Li from Top Green Group (Beijing, China) for providing red clover seeds. We acknowledge Ying Wang, Jun Chen, Fei Wang and Erjun Qin from Novogene Corporation (Beijing, China) for the facilities and expertise of the PacBio platform for libraries construction and sequencing.

\section{Funding}

The program was supported by the National Natural Science Foundation of China (No. 31601989 and No. 31672477). Each of the funding bodies granted the funds based on a research proposal. They had no influence over the experimental design, data analysis or interpretation, or writing the manuscript.

\section{Availability of data and materials}

All relevant supplementary data is provided within this manuscript as Supplementary files. We deposited the raw bam files in the Sequence Read Archives (SRA) of the National Center for Biotechnology Information (NCBI) under SRA accession SRP149129. The Illumina RNA-Seq data was downloaded from SRA under accession number SRR7226326, SRR7226327 and SRR7226324 of Bioproject PRJNA473388. Genomic sequences and gene annotation information of red clover presented in this report are downloaded online (https://doi.org/10.5281/zenodo.17232)

Authors' contributions

L. H. and L. X. conceived and designed the research. Y. C. and J. Y. conducted experiments. S. L. and S. J. analyzed data. Y. C. wrote the manuscript. All authors read and approved the final manuscript.

Ethics approval and consent to participate Not applicable.

Consent for publication

Not applicable.

Competing interests

The authors declare that they have no competing interests. 


\section{Publisher's Note}

Springer Nature remains neutral with regard to jurisdictional claims in published maps and institutional affiliations.

Received: 9 June 2018 Accepted: 19 November 2018

Published online: 26 November 2018

\section{References}

1. Sullivan ML, Quesenberry KH. Red clover (Trifolium pratense). Methods Mol Biol. 2006;343:369-83.

2. Yeung KS, Gubili J. Red clover (Trifolium pratense). J Soc Integr Oncol. 2008; 6(4):176-7.

3. Taylor NL, Quesenberry KH. Red clover science. Dordrecht ; Boston: Kluwer Academic Publishers; 1996

4. Istvanek J, Jaros M, Krenek A, Repkova J. Genome assembly and annotation for red clover (Trifolium pratense; Fabaceae). Am J Bot. 2014;101(2):327-37.

5. Yates SA, Swain MT, Hegarty MJ, Chernukin I, Lowe M, Allison GG, Ruttink T, Abberton MT, Jenkins G, Skot L. De novo assembly of red clover transcriptome based on RNA-Seq data provides insight into drought response, gene discovery and marker identification. BMC Genomics. 2014;15:453.

6. Chakrabarti M, Dinkins RD, Hunt AG. De novo transcriptome assembly and dynamic spatial gene expression analysis in red clover. Plant Genome. 2016;9(2).

7. Zhu FY, Chen MX, Ye NH, Shi L, Ma KL, Yang JF, Cao YY, Zhang YJ, Yoshida $T$, Fernie $A R$, et al. Proteogenomic analysis reveals alternative splicing and translation as part of the abscisic acid response in Arabidopsis seedlings. Plant J. 2017:91(3):518-33.

8. Sharon D, Tilgner $H$, Grubert F, Snyder M. A single-molecule long-read survey of the human transcriptome. Nat Biotechnol. 2013;31(11):1009.

9. Abdel-Ghany SE, Hamilton M, Jacobi JL, Ngam P, Devitt N, Schilkey F, BenHur A, Reddy AS. A survey of the sorghum transcriptome using singlemolecule long reads. Nat Commun. 2016;7:11706.

10. Wang B, Tseng E, Regulski M, Clark TA, Hon T, Jiao Y, Lu Z, Olson A, Stein JC, Ware $D$. Unveiling the complexity of the maize transcriptome by singlemolecule long-read sequencing. Nat Commun. 2016;7:11708.

11. Chen SY, Deng FL, Jia XB, Li C, Lai SJ. A transcriptome atlas of rabbit revealed by PacBio single-molecule long-read sequencing. Sci Rep. 2017;7.

12. Wang $T$, Wang $H$, Cai D, Gao Y, Zhang H, Wang Y, Lin C, Ma L, Gu L. Comprehensive profiling of rhizome-associated alternative splicing and alternative polyadenylation in moso bamboo (Phyllostachys edulis). Plant J. 2017;91(4):684-99.

13. Workman RE, Myrka AM, Wong GW, Tseng E, Welch KC, Timp W. Singlemolecule, full-length transcript sequencing provides insight into the extreme metabolism of the ruby-throated hummingbird Archilochus colubris. GigaScience. 2018;7(3):1-12

14. Gao S, Ren YP, Sun Y, Wu ZF, Ruan JS, He BJ, Zhang T, Yu X, Tian XX, Bu WJ. PacBio full-length transcriptome profiling of insect mitochondrial gene expression. RNA Biol. 2016;13(9):820-5.

15. Dong L, Liu H, Zhang J, Yang S, Kong G, Chu JS, Chen N, Wang D. Singlemolecule real-time transcript sequencing facilitates common wheat genome annotation and grain transcriptome research. BMC Genomics. 2015;16:1039.

16. Hackl T, Hedrich R, Schultz J, Forster F. Proovread: large-scale high-accuracy PacBio correction through iterative short read consensus. Bioinformatics. 2014;30(21):3004-11.

17. Wang B, Regulski M, Tseng E, Olson A, Goodwin S, McCombie WR, Ware D. A comparative transcriptional landscape of maize and sorghum obtained by single-molecule sequencing. Genome Res. 2018;28(6):921-32.

18. Liu X, Mei W, Soltis PS, Soltis DE, Barbazuk WB. Detecting alternatively spliced transcript isoforms from single-molecule long-read sequences without a reference genome. Mol Ecol Resour. 2017;17(6):1243-56.

19. Li Y, Dai C, Hu C, Liu Z, Kang C. Global identification of alternative splicing via comparative analysis of SMRT- and Illumina-based RNA-seq in strawberry. Plant J. 2017;90(1):164-76.

20. Wu TD, Watanabe CK. GMAP: a genomic mapping and alignment program for mRNA and EST sequences. Bioinformatics. 2005;21(9):1859-75.

21. Trapnell C, Roberts A, Goff L, Pertea G, Kim D, Kelley DR, Pimentel H, Salzberg SL, Rinn JL, Pachter L. Differential gene and transcript expression analysis of RNA-seq experiments with TopHat and cufflinks. Nat Protoc. 2012;7(3):562-78.
22. Alamancos GP, Pagès A, Trincado JL, Bellora N, Eyras E. Leveraging transcript quantification for fast computation of alternative splicing profiles. RNA. 2015;21(9):1521-31.

23. Tatusov RL, Galperin MY, Natale DA, Koonin EV. The COG database: a tool for genome-scale analysis of protein functions and evolution. Nucleic Acids Res. 2000;28(1):33-6.

24. Kanehisa M, Goto S, Kawashima S, Okuno Y, Hattori M. The KEGG resource for deciphering the genome. Nucleic Acids Res. 2004;32(Database issue): D277-80.

25. Xie C, Mao X, Huang J, Ding Y, Wu J, Dong S, Kong L, Gao G, Li CY, Wei L. KOBAS 2.0: a web server for annotation and identification of enriched pathways and diseases. Nucleic Acids Res. 2011;39(Web Server issue):W316-22.

26. Eddy SR. Profile hidden Markov models. Bioinformatics. 1998;14(9):755-63.

27. Shimizu K, Adachi J, Muraoka Y. ANGLE: a sequencing errors resistant program for predicting protein coding regions in unfinished cDNA. J Bioinforma Comput Biol. 2006;4(3):649-64.

28. Zheng $Y$, Jiao C, Sun H, Rosli HG, Pombo MA, Zhang P, Banf M, Dai X, Martin GB, Giovannoni JJ, et al. iTAK: a program for genome-wide prediction and classification of plant transcription factors, transcriptional regulators, and protein kinases. Mol Plant. 2016;9(12):1667-70.

29. Jin J, Tian F, Yang DC, Meng YQ, Kong L, Luo J, Gao G. PlantTFDB 4.0: toward a central hub for transcription factors and regulatory interactions in plants. Nucleic Acids Res. 2017:45(D1):D1040-5.

30. Kong L, Zhang Y, Ye ZQ, Liu XQ, Zhao SQ, Wei L, Gao G. CPC: assess the protein-coding potential of transcripts using sequence features and support vector machine. Nucleic Acids Res. 2007;35:W345-9.

31. Sun L, Luo H, Bu D, Zhao G, Yu K, Zhang C, Liu Y, Chen R, Zhao Y. Utilizing sequence intrinsic composition to classify protein-coding and long noncoding transcripts. Nucleic Acids Res. 2013;41(17):e166.

32. Finn RD, Coggill P, Eberhardt RY, Eddy SR, Mistry J, Mitchell AL, Potter SC, Punta M, Qureshi M, Sangrador-Vegas A, et al. The Pfam protein families database: towards a more sustainable future. Nucleic Acids Res. 2016;44(D1): D279-85.

33. Wright MW. A short guide to long non-coding RNA gene nomenclature. Hum Genomics. 2014;8:7.

\section{Ready to submit your research? Choose BMC and benefit from:}

- fast, convenient online submission

- thorough peer review by experienced researchers in your field

- rapid publication on acceptance

- support for research data, including large and complex data types

- gold Open Access which fosters wider collaboration and increased citations

- maximum visibility for your research: over $100 \mathrm{M}$ website views per year

At BMC, research is always in progress.

Learn more biomedcentral.com/submission 